\title{
The Specific Heat of a Trapped Fermi Gas: an Analytical Approach
}

\author{
José M. B. Noronha*, David J. Toms ${ }^{\dagger}$ \\ Department of Physics, University of Newcastle upon Tyne, \\ Newcastle upon Tyne, NE1 7RU, U. K.
}

(October 25, 2018)

\begin{abstract}
We find an analytical expression for the specific heat of a Fermi gas in a harmonic trap using a semi-classical approximation. Our approximation is valid for $k_{B} T \gtrsim \hbar w_{x, y, z}$ and in this range it is shown to be highly accurate. We comment on the semi-classical approximation, presenting an explanation for this high accuracy.
\end{abstract}

${ }^{*}$ Corresponding author. E-mail:J.M.B.D.Noronha@ncl.ac.uk

${ }^{\dagger}$ E-mail:D.J.Toms@ncl.ac.uk 
Trapped ultra-cold Fermi gases have received a lot of attention in the last few years. This was partly triggered by the first observations of Bose-Einstein condensation in 1995 113. Since then, the field has vastly expanded. The trapping and cooling of fermions is in a much less developed state than that of bosons but it will probably not be long before quantum degeneracy is achieved [4]. (For recent experiments see [5],6].)

On the theoretical side, Butts and Rokhsar [7] have calculated the specific heat numerically and provided analytical results for the spatial and momentum distribution of a non-self-interacting spin polarised Fermi gas at $T=0$ in a harmonic trap using the semiclassical (Thomas-Fermi) approximation. Their results are valid for large particle numbers. In addition, Schneider and Wallis [8] made a numerical study of a similar gas and focused on the effects of small particle numbers. The present paper complements that of Butts and Rokhsar in that we provide an analytical expression for the specific heat of a non-selfinteracting spin polarised Fermi gas in the context of a semi-classical approximation. We then compare it to the exact result given by a numerical calculation. As we shall see, the semi-classical approximation produces extremely good results for $k T \gtrsim \hbar w_{x, y, z}$, where $w_{x, y, z}$ are the frequencies of the trap in the three spatial directions, for particle numbers as low as $N=1000$. This high level of accuracy is perhaps unexpected. We will discuss this and an explanation will be given. The characteristic temperature for quantum degeneracy, the Fermi temperature, is given by $k T_{F}=\hbar w(6 N)^{\frac{1}{3}}$ for an isotropic trap. The condition we impose is therefore valid for a quantum degenerate regime. It is also worth noting that this condition holds in current experiments with trapped fermions [6].

The effects of interactions has been dealt with in several works [9 [16] and in particular the possibility of a BCS transition has been studied in the case of ${ }^{6} \mathrm{Li} 9$ 11]. Considering the gas to be non-interacting is a very good approximation for a dilute neutral atomic gas. The only case where interactions could be important is trapped ${ }^{6} \mathrm{Li}$ with two hyper-fine spin states [12]. ${ }^{6} \mathrm{Li}$ has an anomalously large s-wave scattering length. At least two different states are needed for s-wave scattering as it is forbidden for particles in the same state. However, the results of ref. [11] are for $k T<<\hbar w$, lying outside the range of temperatures concerned in the present work.

Consider a gas of fermions in a harmonic potential. Neglecting interactions between the particles, the energy levels of each particle are

$$
\begin{aligned}
E_{n_{1} n_{2} n_{3}} & =\left(n_{1}+\frac{1}{2}\right) \hbar w_{1}+\left(n_{2}+\frac{1}{2}\right) \hbar w_{2}+\left(n_{3}+\frac{1}{2}\right) \hbar w_{3}, \\
n_{i} & =0,1,2, \ldots
\end{aligned}
$$

where $w_{1,2,3}$ are the frequencies of the trap.

We will use grand-canonical statistics throughout. This makes the calculations much easier and is justified as the difference between canonical and grand-canonical results is minute for the particle numbers we consider [8].

The number of particles in the system is

$$
N=\sum_{n}\left[e^{\beta\left(E_{n}-\mu\right)}+1\right]^{-1} .
$$

The internal energy of the system is given by 


$$
U=\sum_{n} E_{n}\left[e^{\beta\left(E_{n}-\mu\right)}+1\right]^{-1} .
$$

$\mu$ is the chemical potential and the sum in (2) and (3) is over all particle states. Here it is convenient for simplicity of the expression, to make the substitutions

$$
\begin{aligned}
w_{1} & =w, \\
w_{2} & =\lambda w \\
w_{3} & =\lambda^{\prime} w, \\
x & =\beta \hbar w, \\
\mu & =\hbar w\left(\frac{1+\lambda+\lambda^{\prime}}{2}-\epsilon\right),
\end{aligned}
$$

where $x, \epsilon, \lambda$ and $\lambda^{\prime}$ are newly defined dimensionless variables. The internal energy becomes

$$
U=\frac{1}{2} \hbar w\left(1+\lambda+\lambda^{\prime}\right) N+\frac{1}{2} \hbar w u
$$

where

$$
\begin{aligned}
& u=2 \sum_{n_{1}=0}^{\infty} \sum_{n_{2}=0}^{\infty} \sum_{n_{3}=0}^{\infty} k\left[e^{x(k+\epsilon)}+1\right]^{-1} \\
& k=n_{1}+\lambda n_{2}+\lambda^{\prime} n_{3} .
\end{aligned}
$$

Only the second term contributes to the specific heat since $N$ is held fixed. We have

$$
C=\left(\frac{\partial U}{\partial T}\right)_{N, w}=\frac{1}{2} \hbar w\left(\frac{\partial u}{\partial T}\right)_{N, w} .
$$

Note that the partial derivative at constant particle number and trap frequency is the natural way of defining the specific heat for a gas in the situation concerned. After a change of variables from $T$ to $x$ this becomes

$$
\frac{C}{k_{B}}=-\frac{1}{2} x^{2}\left(\frac{\partial u}{\partial x}\right)_{N, w},
$$

where $k_{B}$ is Boltzmann's constant.

The simplest approximation consists in replacing the triple sums in (6) by a triple integral. Thus,

$$
u \simeq v=2 \int_{0}^{+\infty} \int_{0}^{+\infty} \int_{0}^{+\infty} k\left[e^{x(k+\epsilon)}+1\right]^{-1} d y_{1} d y_{2} d y_{3}
$$

and $k$ is now the real function $k=y_{1}+\lambda y_{2}+\lambda^{\prime} y_{3}$. Changing the integrating variable to $k$ we have

$$
v=\frac{1}{\lambda \lambda^{\prime}} \int_{0}^{+\infty} k^{3}\left[e^{x(k+\epsilon)}+1\right]^{-1} d k
$$

Note that for the isotropic case we could have transformed the triple sum in (6) into a single sum and only then convert it to an integral, yielding 


$$
v=\frac{1}{\lambda \lambda^{\prime}} \int_{0}^{+\infty}\left(k^{3}+3 k^{2}+2 k\right)\left[e^{x(k+\epsilon)}+1\right]^{-1} d k,
$$

which, at first sight, might be thought to be a more accurate expression. For the anisotropic case, though not trivial, we could likewise have a density of states expanded to three terms [17,18. We will coment on this shortly.

To solve the integral in (10) we Taylor expand the integrand in powers of $e^{x(k+\epsilon)}$ and integrate each resulting term. Here we have to consider two different cases: $\epsilon>0$ and $\epsilon<0$. For $\epsilon>0$ this yields

$$
v=\frac{6}{\lambda \lambda^{\prime} x^{4}} \sum_{n=1}^{\infty}(-1)^{n+1} \frac{e^{-n x \epsilon}}{n^{4}} .
$$

From (8) and (12) and retaining the fact that $v$ is an approximated version of $u$, we have

$$
\frac{C}{k_{B}} \simeq \frac{12}{\lambda \lambda^{\prime} x^{3}} A_{4}+\frac{3}{\lambda \lambda^{\prime} x^{2}}\left(\frac{\partial(\epsilon x)}{\partial x}\right)_{N, w} A_{3}
$$

where $A_{i}$ denotes the sum

$$
A_{i}=\sum_{n=1}^{\infty}(-1)^{n+1} \frac{e^{-n x|\epsilon|}}{n^{i}} .
$$

The use of the absolute value of $\epsilon$ is redundant in the case we are considering $(\epsilon>0)$ but it is useful when we consider the $\epsilon<0$ case.

To obtain an expression for the partial derivative in (13) we use the equality

$$
\left(\frac{\partial N}{\partial x}\right)_{N, w}=0
$$

with $N$, the number of particles, being

$$
N \simeq \frac{A_{3}}{\lambda \lambda^{\prime} x^{3}}
$$

Expression (16) is obtained using the same process as was used to obtain (13). We then have

$$
\left(\frac{\partial(\epsilon x)}{\partial x}\right)_{N, w} \simeq-\frac{3}{x} \frac{A_{3}}{A_{2}}
$$

and

$$
\frac{C}{k_{B}} \simeq \frac{3}{\lambda \lambda^{\prime} x^{3}}\left(4 A_{4}-3 \frac{A_{3}^{2}}{A_{2}}\right)
$$

Finally we note that the sums $A_{i}$ can be put in terms of polylogarithms [19], yielding

$$
\lambda \lambda^{\prime} \frac{C}{k_{B}} \simeq 3 x^{-3}\left[4 L i_{4}\left(e^{-x \epsilon}\right)-\frac{1}{2} L i_{4}\left(e^{-2 x \epsilon}\right)-3 \frac{\left[L i_{3}\left(e^{-x \epsilon}\right)-\frac{1}{4} L i_{3}\left(e^{-2 x \epsilon}\right)\right]^{2}}{L i_{2}\left(e^{-x \epsilon}\right)-\frac{1}{2} L i_{2}\left(e^{-2 x \epsilon}\right)}\right] .
$$


For $\epsilon<0$, the procedure is similar but slightly more complicated. The integral of expression (10) must be divided in two parts, according to whether the exponential is greater or smaller than 1, as the Taylor expansion of the integrand is different for the two cases. The final result for $\epsilon<0$ is

$$
\lambda \lambda^{\prime} \frac{C}{k_{B}} \simeq-12 A_{4} x^{-3}+21 \zeta(4) x^{-3}+6 \zeta(2) \epsilon^{2} x^{-1}+\frac{1}{2} \epsilon^{4} x+\frac{\left(A_{3}-\zeta(2) \epsilon x-\frac{1}{6} \epsilon^{3} x^{3}\right)^{2}}{A_{2}-\zeta(2)-\frac{1}{2} \epsilon^{2} x^{2}},
$$

where $\zeta$ is the Riemann zeta function. Putting the sums $A_{i}$ in terms of polylogarithms we have

$$
\begin{aligned}
\lambda \lambda^{\prime} \frac{C}{k_{B}} \simeq & {\left[-12 L i_{4}\left(e^{x \epsilon}\right)+\frac{3}{2} L i_{4}\left(e^{2 x \epsilon}\right)+21 \zeta(4)\right] x^{-3}+6 \zeta(2) \epsilon^{2} x^{-1}+\frac{1}{2} \epsilon^{4} x+} \\
& \frac{\left[L i_{3}\left(e^{x \epsilon}\right)-\frac{1}{4} L i_{3}\left(e^{2 x \epsilon}\right)-\zeta(2) \epsilon x-\frac{1}{6} \epsilon^{3} x^{3}\right]^{2}}{L i_{2}\left(e^{x \epsilon}\right)-\frac{1}{2} L i_{2}\left(e^{2 x \epsilon}\right)-\zeta(2)-\frac{1}{2} \epsilon^{2} x^{2}} .
\end{aligned}
$$

Note that these analytical expressions are not an approximation over the Thomas-Fermi result. They are the exact Thomas-Fermi result, which of course, is in itself an approximation. We computed the specific heat numerically using an exact expression composed of several sums, which is easily obtained inserting (6) in (8) and using (15) to get an expression for $(\partial(\epsilon x) / \partial x)_{N, w}$. This is then compared to the values for the specific heat given by (18) and (20). The accuracy of the Thomas-Fermi approximation is extremely good for $x$ of order 1 or less, as can be seen in figures 1 and 2. As an example, for $N=1000$ and $x_{1}=x_{2}=x_{3}<2.5$ the error in $C$ is always less than $1 \%$ and if we take $x_{i}<1$, the error is always less than $0.1 \%$. For larger values of $x$ the approximation rapidly deteriorates. The results for a higher number of particles are even more accurate, as expected from this kind of approximation []].

Given that for this approximation we have replaced triple sums by triple integrals, such a level of accuracy is quite surprising. Note that the expression inside the sums in (6) falls off exponentially. Therefore the first few terms are very important and expression (9) should differ significantly from (6). And indeed it does. The reason our results are so accurate is that the value of $\epsilon$ used in (18) and (20) is not the same as the one used in (6). For our Thomas-Fermi approximation we took $\epsilon$ from expression (16), ie, an approximate $\epsilon$. So, the insertion of an approximate $\epsilon$ in an approximate expression leads to a fortunate cancelation of errors. We have tested this by inserting the exact value of $\epsilon$ into (18) and (20). This leads to much worse results. Also, we have tried to further improve our approximation by considering the first two terms of the cubic polynomial in (11) instead of only the first term and doing the same to the number of particles, obtaining an additional term in (16) as well as additional terms in (18) and (20). To our surprise, this only increases the error. We can only assume that the very high level of accuracy of the Thomas-Fermi approximation is a happy coincidence. In fact, if we take the exact value of $\epsilon$ and input it in expressions (18) and (20) and in the equivalent expressions obtained using $k^{3}+3 k^{2}$ in (11) instead of only $k^{3}$, then the latter yield the most accurate result, though still not a very good one.

\section{ACKNOWLEDGMENTS}

J.N. acknowledges financial support by the Portuguese Foundation for Science and Technology under grant PraxisXXI/ BD/5660/95. 


\section{REFERENCES}

[1] M. H. Anderson, J. R. Ensher, M. R. Matthews, C. E. Wieman and E. A. Cornell, Science, 269 (1995) 198

[2] C. C. Bradley, C. A. Sackett, J. J. Tollett and R. G. Hulet, Phys. Rev. Lett., 75 (1995) 1687

[3] K. B. Davis, M.-O. Mewes, M. R. Andrews, N. J. van Druten, D. S. Durfee, D. M. Kurn and W. Ketterle, Phys. Rev. Lett., 75 (1995) 3969

[4] M. J. Holland, B. DeMarco and D. S. Jin, preprint cond-mat/9911017

[5] F. S. Cataliotti, E. A. Cornell, C. Fort, M. Inguscio, F. Marin, M. Prevedelli, L. Ricci and G. M. Tino, Phys. Rev. A, 57 (1998) 1136

[6] B. DeMarco, J. L. Bohn, J. P. Burke, Jr., M. Holland and D. S. Jin, Phys. Rev. Lett., 82 (1999) 4208

[7] D. A. Butts and D. S. Rokhsar, Phys. Rev. A, 55 (1997) 4346

[8] J. Schneider and H. Wallis, Phys. Rev. A, 57 (1998) 1253

[9] H. T. C. Stoof, M. Houbiers, C. A. Sackett and R. G. Hulet, Phys. Rev. Lett., 76 (1996) 10

[10] M. Houbiers, R. Ferwerda, H. T. C. Stoof, W. I. McAlexander, C. A. Sackett and R. G. Hulet, Phys. Rev. A, 56 (1997) 4864

[11] H. T. C. Stoof and M. Houbiers, Proceedings of the International Summer School "Enrico Fermi" on Bose-Einstein condensation in atomic gases, Varenna 1998, edited by M. Inguscio, S. Stringari and C. E. Wieman

[12] G. M. Bruun and K. Burnett, Phys. Rev. A, 58 (1998) 2427

[13] F. Brosens, J. T. Devreese and L. F. Lemmens, Phys. Rev. E, 57 (1998) 3871

[14] F.Brosens, J. T. Devreese and L. F. Lemmens, Phys. Rev. E, 58 (1998) 1634

[15] G. Bruun, Y. Castin, R. Dum and K. Burnett, Euro. Phys. J. D, 7 (1999) 433

[16] M. Amoruso, I. Meccoli, A. Minguzi and M. P. Tosi, preprint cond-mat/9909102

[17] K. Kirsten and D. J. Toms, Phys. Lett. A, 222 (1996) 148

[18] S. Grossman, M. Holthaus, Phys Lett. A, 208 (1995) 188

[19] K. Kirsten and D. J. Toms, Phys. Rev. A, 54 (1996) 4188 


\section{FIGURES}

FIG. 1. the familiar curve of the specific heat ploted against $x=\hbar w / k_{B} T$ for $\lambda=\lambda^{\prime}=1$

(isotropic harmonic oscilator) and $N=1000$. Both the analytical and numerical results are shown, but they are so similar that it looks like one curve only.

FIG. 2. the ratio $C_{\text {analytical }} / C_{\text {numerical }}$ ploted against $x$ for $\lambda=\lambda^{\prime}=1$ and $N=1000$ 
fig. 1

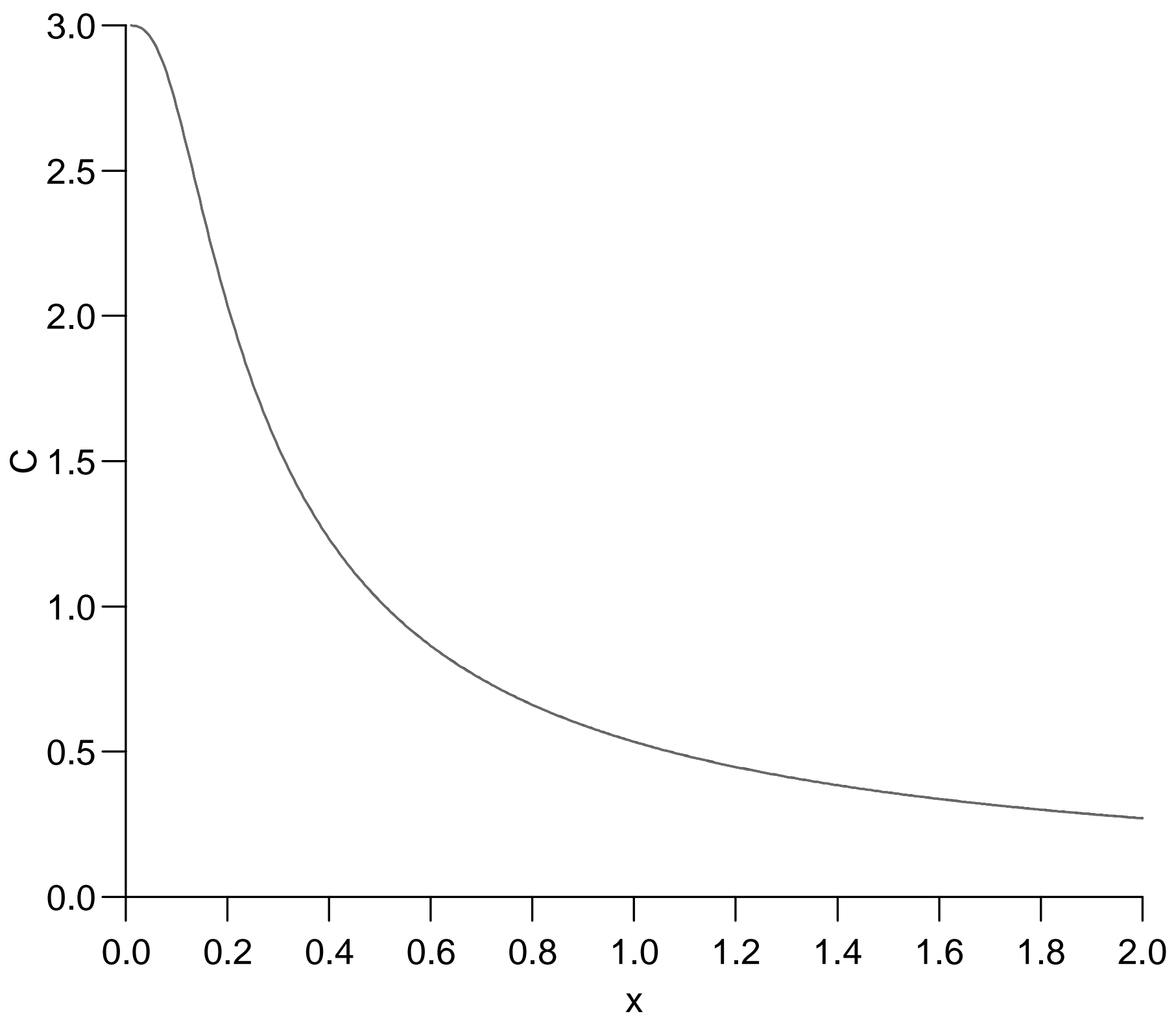


fig.2

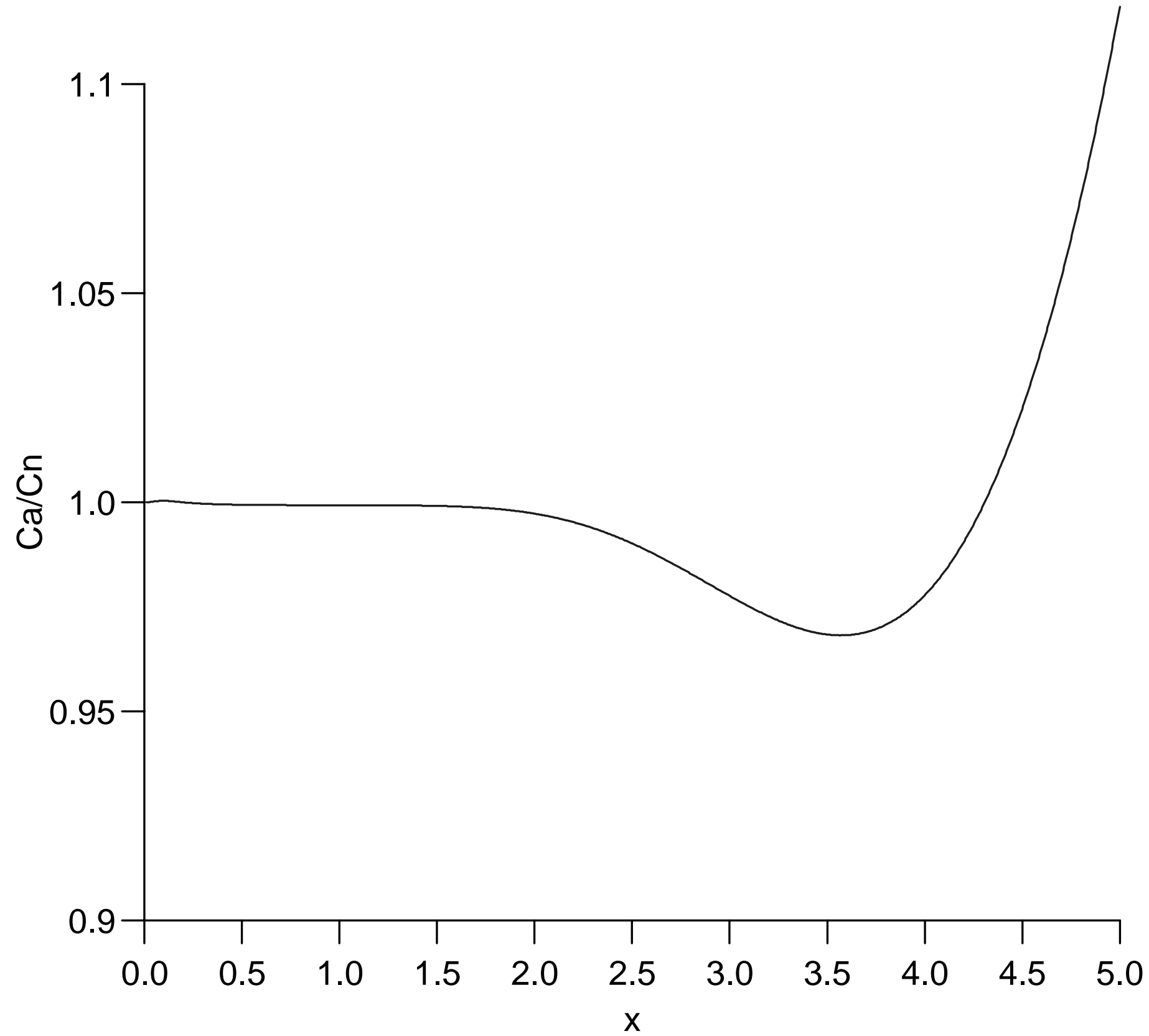

